\title{
Zur Kenntnis der Säureintoxikation.
}

\section{Von}

Gertrude D. Bostock, M. B., B. Sc. Carnegie Fellow.

(Aus der chemitchen Abteilung des pathologiachen Instituts der Universitat Berlin.) (Der Redaktion zugegangen am 28. März 1913.)

Vor längerer Zeit fand E. Salkowski,(1) daß Kaninchen an eingegebene Säuren, sowie an die aus eingeführtem Taurin gebildeten Schwefelsäure bei «saurer Nahrung, Alkali von ihrem Körper abgeben und dabei zugrunde gehen, augenscheinlich an der Alkalientziehung, während Hunde nach den Versuchen von

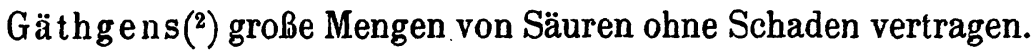

Damit war ein wichtiger Unterschied in dem Chemismus des Körpers zwischen Herbivoren und Carnivoren festgestellt. Die Ursache, warum sich Herbivoren und Carnivoren so verschieden verhalten, wurde erst von Schmiedeberg und Walter ${ }^{(3)}$ aufgedeckt. Sie fanden, daß die Carnivoren die zugeführte Säure durch neugebildetes Ammoniak neutralisieren,*) während Herbivoren hierzu nicht imstande zu sein scheinen. Walter gelang es auch, Kaninchen, die infolge ron Säurezufuhr im Sterben lagen, durch Injektion von Alkali in die Venen am Leben zu erhalten. Damit war der Beweis erbracht, daß in der Tat nur die Alkalientziehung die Ursache des Säuretodes war. Walter stellte ferner die tödliche Dosis auf $0,9 \mathrm{~g}$ $\mathrm{HCl}$ pro Kilo Tier fest, , wenn diese Quantität Säure, natürlich stark verdünnt, innerhalb 24 Stunden per os gegeben wurde:

Im Jahre 1906 hat nun Eppinger $\left(^{(}\right)$die Resultate einiger Versuche an Kaninchen veröffentlicht, welchen die Idee zugrunde liegt, daß es möglich sein möchte, auch Kaninchen vor der tödlichen Wirkung der Säure zu schützen, wenn man ihnen gleichzeitig mit der Säure Substanzen zuführt, aus welchen eventuell eine Abspaltung von Ammoniak zu erwarten war. Eppinger hat die Säuredosis von Walter benützt. Er gab seinen Kaninchen $100 \mathrm{ccm}$ n/4-Salzsäure $(=0,9125 \mathrm{~g} \mathrm{HCl})$ pro

*) Eine dahingehende Vermutung hat auch Salkowski l. c. schon ausgesprochen. 
Kilo Körpergewicht und beobachtete, daß die Tiere regelmäßig nach dieser Dosis innerhalb 22 bis 24 Stunden starben, daß dagegen ein Kaninchen, wenn man ihm Glykokoll, Alanin, Asparaginsäure oder Harnstoff subcutan injiziert und gleichzeitig die Säure per os verabreicht, nicht stirbt und den Tag nach der letzten Einspritzung vollkommen gesund zu. sein scheint. Anderseits sind Acetamid, Formamid und Witte-Pepton nicht imstande, das Tier gegen Säurevergiftung zu schützen.

In den Versuchen VI und. VII von Eppinger, in denen jedësmal je $5 \mathrm{~g}$ Glykokoll gleichzeitig mit der Säurezufuhr gegeben wurde, zeigte eine Harnanalyse, daß das Ammoniak sich nur mit ungefähr einem Drittel der ausgeschiedenen Säure verbunden hatte; während die übrigen zwei Drittel an fixes Alkali gebunden waren.

Pohl und Münzer $\left(^{4}\right)$ wiederholten einige Versuche Eppingers mit Harnstoff und Glykokoll und konnten seine Resultate nicht bestätigen. Sie konnten absolut nichts von irgend einem schützenden Einfluß gegen Säurevergiftung feststellen. Wenn diese Einspritzungen überhaupt einen Einfluß hatten, dann beschleunigten sie eher den Tod der Tiere, anstatt ihn zu verhindern. Auf Veranlassung von Prof. E. Salkowski habe ich neue Versuche über diese Frage angestellt, über die im folgenden berichtet werden soll.

Anordnung der Versuche.

Die Tiere wurden mit Hafer gefüttert, um eine saure Reaktion des Harns hervorzurufen, da die schädigende Wirkung, zugeführter Säure sich natürlich nur bei einer asauren Nahrung * geltend machen kann.

Der Versuch wurde begonnen, sobald der Harn sauer reagierte, d. h. gewöhnlich nach zwei Tagen nach Beginn der Haferfütterung. Die ersten zwei Tage erhielt das Tier $100 \mathrm{ccm}$ destilliertes Wasser mittels Schlundsonde, am dritten Tage wurde dem Tier vormittags und nachmittags je ein Drittel der tödlichen Säuredosis verabreicht. Am vierten Tag wurde das Tier in gleicher Weise behandelt, $d$. h., wenn es nach der dritten Dosis nicht gestorben war, bekam es eine vierte. 
Die benützte Säure war $n / 1-\mathrm{HCl}$ und die tödliche Dosis war genau dieselbe wie bei Eppingers Versuchen, also $100 \mathrm{ccm} \mathrm{n/4-HCl}$ pro Kilo Körpergewicht. Die Säure war jedesmal stark verdünnt bis $120-150 \mathrm{ccm}$.

In den ersten zwei Versuchen (I und II) wurde Säure allein verabreicht; da keines von beiden Tieren innerhalb $41 / 2$ bis 5 Stunden nach dem letzten Teil der «tödlichen. Dosis gestorben war, wurde ihnen noch eine vierte Dosis gegeben.

In Versuch III und IV suchte ich die Schutzwirkung von Glykokoll festzustellen. Gleichzeitig mit jeder Säuredosis bekam das Tier eine subcutane Einspritzung von $4 \mathrm{~g}$ Glykokoll. Beide Tiere starben, Nr. III in der Nacht nach der letzten Säuredosis: Nr. IV dagegen erst 48 Stunden nach der letzten Dosis, allerdings sah es während dieser Zeit sehr krank aus.

Wenn die Aminogruppe des Glykokolls einen schützenden Einfluß hätte, dann müßte man ebenso einen schützenden Einfluß von den organischen Salzen des Ammoniaks erwarten. In dieser Erwägung erhielten die Tiere V, VI und VII eine Lösung, die 0,5 g essigsaures Ammoniak enthielt, gleichzeitig mit der Säure mittels Schlundsonde. Nr. V starb gleich nach der dritten Dosis infolge versehentlicher Einführung der Schlundsonde in die Trachea.

Nr. VI und VII starben nach der dritten Dosis.

Der Harn der zwei Tage vor der Säurezufuhr wurde zur Analyse vereinigt. In der Tabelle ist das Mittel von diesen zwei Tagen angegeben. Der Harn eines jeden Säuretages wurde einzeln analysiert. Die Menge des zweiten Säuretages entspricht nicht ganz derjenigen von 24 Stunden, da die Tiere meistens während der Nacht starben.

Die Harnblase wurde jeden Vormittag ausgedrückt, um den Harn der einzelnen Tage möglichst gut von einander abzugrenzen.

Die Analyse beschränkte sich auf die Bestimmung des Totalstickstoffs nach Kjeldahl und des Ammoniaks nach Krüger-Reich-Schittenhelm, da die Frage, um wieviel die Ausscheidung von Ammoniak sich durch Säurezufuhr vermehrt, am wichtigsten war. 
Nachfolgende Tabelle ${ }^{1}$ ) enthält die gefundenen Resultate.

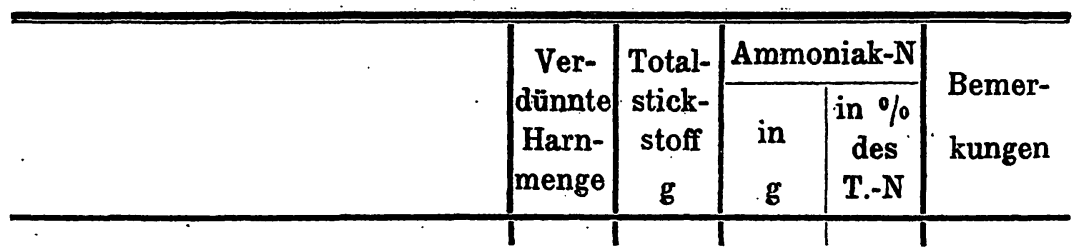

Versuch $\mathrm{I}$.

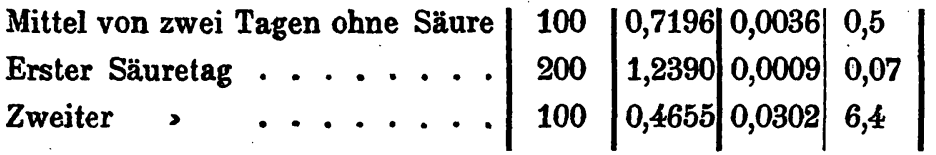

Versuch II.

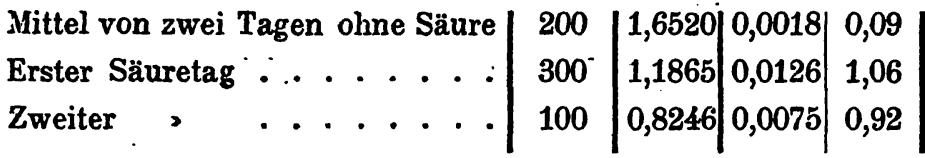

Versuch III.

Mittel von zwei Tagen ohne Säure |

Erster Säure- und Glykokolltag .

400

Zweiter

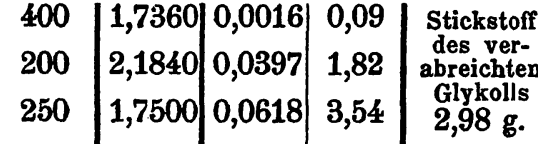

Versuch IV.

Mittel von zwei Tagen ohne Säure |

Erster Säure- und Glykokolltag .

Zweiter Säuretag . . . ...

\begin{tabular}{l|l|l|l|}
300 & 1,0605 & 0,0050 & 0,47 \\
400 & 2,7440 & 0,0851 & 3,1 \\
400 & 2,6880 & 0,1759 & 6,58 \\
300 & 1,2600 & 0,0685 & 5,4
\end{tabular}

do.

Versuch V.

\begin{tabular}{l|l|l|l|l|l} 
Mittel von zwei Tagen ohne Säure & 125 & 0,6212 & 0,0045 & 0,73 & $\begin{array}{c}\text { Stickstoff } \\
\text { des zuge- } \\
\text { fuhrten Am- } \\
\text { monacetats } \\
0,2727 \mathrm{~g} .\end{array}$ \\
BeiSäure-und Ammonacetatzufuhr & 200 & 0,9520 & 0,0259 & 2,7 &
\end{tabular}

Versuch VI.

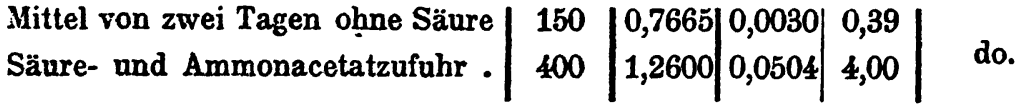

Versuch VII.

\begin{tabular}{c|c|c|c|c|c} 
Mittel von zwei Tagen ohne Săure & 250 & 0,7568 & 0,0022 & 0,3 \\
Säure- und Ammonacetafzufuhr. & 300 & 1,3860 & 0,1314 & 9,4 \\
$, \quad, \quad$ & 280 & 0,5512 & 0,0464 & 8,4
\end{tabular} \mid do.

1) Die Beschreibung der einzelnen Versuche siehe weiter unten. 


\section{Erörterung der Resultate.}

Es ist eine interessante Tatsache, daß keines von meinen beiden Kaninchen innerhalb $4^{1} / 2$ bis 5 Stunden nach der tödlichen Säuredosis gestorben ist, Eppinger gibt an, daß die seinen innerhalb 2 bis 21/2 Stunden nach der letzten Dosis gestorben sind. Anscheinend besteht ein erheblicher Unterschied in der Widerstandskraft verschiedener Tiere. Nun wurden zwar meine Tiere mit Hafer, die von Eppinger mit Grünfutter gefüttert; wenn aber das Futter überhaupt irgend einen Einfluß auf den früheren oder späteren Eintritt der Vergiftung ausübt, wäre zu erwarten gewesen, daß die Säurevergiftung bei dem mit Grünfutter gefütterten Tiere später als bei dem mit Hafer gefütterten eintritt. In beiden Versuchen (I und In) stieg die Gesamtmenge des ausgeschiedenen Ammoniaks an den Säuretagen, und zwar in I mehr als in II, wahrscheinlich liegt die Erklärung in der Tatsache, daß Kaninchen II gleich nach der vierten Dosis starb und deshalb nicht so viel Harn ausscheiden konnte. In keiner Weise genügt die Menge von Ammoniak, die Totalmenge der verabreichten Säure zu binden, wie nach den von Salko w ski ausgeführten Harnanalysen bei Säurekaninchen, bei denen sämtliche Säuren und Basen des Harns bestimmt und fast völlige Äquivalenz gefunden wurde, auch nicht anders zu erwarten war. Das Ammoniak, in Prozenten vom Totalstickstoff berechnet, ist an den Säuretagen bedeutend vermehrt. Gleichzeitige subcutane Einspritzung von Glykokoll hat die Tiere nicht gerettet, doch blieb $\mathrm{Nr}$. IV verhältnismäßig länger am Leben als die anderen, d. h. 48 Stunden. Die Totalmenge des ausgeschiedenen Ammoniaks war an den Säuretagen erheblich vermehrt und zwar weitaus mehr als bei den Tieren I und II. Merkwürdigerweise war bei Nr. IV die Menge des Ammoniaks an dem auf den Säuretag folgenden Tage sehr groß. Auch der Prozentgehalt des Ammoniaks ist bedeutend höher. Offenbar hat also das Glykokoll hier Ammoniak zur Neutralisierung der Säure geliefert. Die gleichzeitige Verabreichung von essigsaurem Ammoniak in den Versuchen V, VI . und VII hat den Tod nicht verhindert, sondern ihn im Gegen- 
teil beschleunigt, wobei die dreifache Drittelḋosis der Säure genügte, statt der sonst angewändten vierfachen.

Die Tiere $\dot{V}$ und VI haben an dem zweiten Säuretag keinen Harn ausgeschieden. Bei der Sektion war die Harnblase leer. Der Prozentgehalt des Ammoniaks wurde an den Säuretagen erheblich vermehrt gefunden.

Es sei bemerkt, daß die von Eppinger beschriebenen Pendelbewegungen des Kopfes nur an dem Tiere VII in der ganzen Reihe beobachtet wurden. Aus den Versuchen kann man wohl schließen, daß eine Vermehrung des verfügbaren Ammoniaks im Organismus eine Säurevergiftung zu verhindern nicht imstande ist. Ich lasse nunmehr die Versuchsprotokolle folgen.

7. 10. 12. Gewicht $2,200 \mathrm{~kg}$.

$$
\text { Versuch I. }
$$

Nach zwei Tagen Haferfütterung reagierte der Harn sauer,

9. 10. 12. $10 \mathrm{Uhr}$ vormittags $100 \mathrm{ccm}$ destilliertes Wasser mittels Schlund-

10. 10. 12. sonde eingeführt.

11. 11. 12. Gewicht $2,100 \mathrm{~kg}$.

$10 \mathrm{Uhr}$ vormittags $17,8 \mathrm{~cm} \mathrm{n} / \mathrm{h}-\mathrm{HCl}$ verdünnt mit destilliertem Wasser bis $120 \mathrm{~cm}$ mittels Schlundsonde eingeführt.

12. 10. 12. 10 > vormittags

4 Uhr 15 nachmittags $\because$ do.

2 . 30 nachmittags

Bemerkungen: Das Tier schien an beiden Sẳuretagen ganz munter zu sein, fraß jedoch nicht. Tod in der Nachit zum 13. 10.

Bei der Sektion wurde nichts Abnormes beobachtet, außer einigen kleinen Ecchỵmosen in der Magenmucosa. Dié Nieren kongestioniert.

Es sei betont, daß das Tier nicht innerhalb $4^{1 / 2}$ Stunden der dritten Säuredosis gestorben ist.

12. 10. 12. Gewicht $2,600 \mathrm{~kg}$ : Haferfütterung zwei Tage.

14. 10. 12. $10 \mathrm{Uhr}$ vormittags $100 \mathrm{ccm}$ destilliertes Wasser mittels Schlundsonde eingeführt.

15. 10. 12. do.

16. 10. 12. Gewicht $\bar{z}, 550 \mathrm{~kg}$.

$10 \mathrm{Uhr}$ vormittags $21,3 \mathrm{ccm} \mathrm{n} / \mathbf{1}-\mathrm{HCl}$ mit destilliertem Wasser verdünnt bis $150 \mathrm{ccm}$ mittels Schlundsonde eingeführt.

3 Uhr nachmittags .

17. 10.12. 10 , vormittags

3 , nachmittags

do. 
Bemerkungen: Das Tier fraß an den Säaretagen nicht. Plötzlicher Tod ungefähr eine Stunde nach der letzten Säuredosis.

Dic Soktion ergab, dab die Magenmucosa infolge der postmortalen Säurewirkung erheblich angedaut war. Die Nieren injiziert.

\section{Versuch III.}

An den Tagen vor der Säurezufuhr war das Tier genau wie in Versuch I und II behandelt worden.

23. 10. 12. Gewicht $2,730 \mathrm{~kg}$.

$10 \mathrm{Uhr}$ vormittags $22,8 \mathrm{ccm} n / 1-\mathrm{HCl}$ verdünnt bis $120 \mathrm{ccm}$ mit destilliertem Wasser mittels Schlundsonde eingeführt. $4 \mathrm{~g}$ Glykokoll in $50 \mathrm{ccm}$ Wasser gelöst, subcutan eingespritzt.

3 Uhr 30 nachmittags do.

24. 10.12. 10 , vormittags

3 , 30 nachmittags

Bemerkungen: Das Tier sieht an dem zweiten Säuretage krank aus. Die Atemzüge wurden vertieft. Das Tier fraß an den beiden Säuretagen nicht. Tod während der Nacht des zweiten Säuretages.

Sektion: eine große hämorrhagische Erosion in Magenmucosa. Nieren injiziert.

Eine Probe der Flüssigkeit des subcutanen Gewebes wurde auf Glykokoll geprüft - es enthielt kein Glykokoll.

\section{Versuch IV.}

An den Tagen vor der Säurezufuhr wurde das Tier genau wie in Versuch I behandelt.

18. 10. 12. Gewicht $3,100 \mathrm{~kg}$.

22. 10.12. > 2,800 ,

$10 \mathrm{Uhr} 30$ vormittags $23,3 \mathrm{ccm}$ n/1-HCl mit destilliertem Wasser bis $150 \mathrm{ccm}$ verdünnt mittels Schlundsonde eingeführt. $4 \mathrm{~g}$ Glykokoll in $50 \mathrm{ccm}$ destillierten Wasser gelöst subcutan eingespritzt.

3 Uhr nachmittags do.

23. 10.12. 10 , vormittags ,

3 , nachmittags ,

24. 10. 12. Das Tier ist noch am Leben, doch ist es sehr krank und hat Dyspnoe.

25. 10. 12. Sehr. krank.

26. 10. 12. Tot.

Sektion: Eine große Erosion in der Magencardia. Nieren injiziert.

Versuch V.

2: 11. 12. Gewicht $2,170 \mathrm{~kg}$.

Behandlung vor der Säurezufuhr wie bei Versuch I.

6. 11. 12. Gewicht $2,130 \mathrm{~kg}$. 
$10 \mathrm{Uhr}$ vormittags $17,7 \mathrm{ccm} \mathrm{n/1-HCl}+0,5 \mathrm{~g}$ essigsaures Ammon mit destilliertem Wasser verdünnt bis $120 \mathrm{ccm}$ mittels Schlundsonde eingeführt.

3 Uhr nachmittags do.

7. 11. 12.10 , vormittags

3 , nachmittags

Bemerkungen: An dem zweiten Säuretag sah das Tier krank aus, starb leider während der letzten Injektion (s. o.)

Sektion: Mehrere kleine Ecchymosen in der Magen- und Darmmucosa.

Versuch VI.

3. 11. 12. Gewicht $2,220 \mathrm{~kg}$.

Behandlung vor der Säurezufuhr genau wie in Versuch I.

7. 11. 12. Gewicht $2,200 \mathrm{~kg}$.

$10 \mathrm{Uhr}$ vormittags $18,25 \mathrm{ccm} \mathrm{n} / \mathrm{s}-\mathrm{HCl}+0,8 \mathrm{~g}$ essigsaures Ammon mit destilliertem Wasser bis $120 \mathrm{ccm}$ verdünnt.

3 Uhr nachmittags do.

8. 11. 12. 10 , vormittags

Bemerkungen: Das Tier sah krank an dem zweiten Säuretage aus und Atmung vertieft. Tot zwei Stunden nach der dritten Säuredosis.

Sektion: Große hämorrhagische Erosion in der Magenmucosa.

Versuch VII.

9. 11. 12. Gewicht 1,970 .

Behandlung vor der Säurezufuhr genau wie in Versuch I.

13. 11. 12. Gewicht $1,730 \mathrm{~kg}$.

$10 \mathrm{Uhr}$ vormittags $14,4 \mathrm{ccm} \mathrm{n} / \mathrm{s}-\mathrm{HCl}+0,5 \mathrm{~g}$ essigsaures Ammon mit destilliertem Wasser bis $120 \mathrm{ccm}$ verdünnt mittels Schlundsonde eingeführt.

$3 \mathrm{Uhr}$ nachmittags do.

14.11.12. 11 , vormittags

Bemerkungen: Das Tier sah an dem zweiten Säuretage krank aus, nach der dritten Dosis wurden Pendelbewegungen des Kopfes beobachtet. Eine vierte Dosis konnte nicht verabreicht werden, weil das Tier am nächsten Tage tot gefunden wurde.

Sektion: Große hämorrhagische Erosion in der Magenmucosa, große hämorrhagische Flecke in den ersten $5 \mathrm{ccm}$ der Duodenummucosa.

Meine Resultate stimmen mit denjenigen von Pohl und Münzer insoweit überein, daß meine Kaninchen durch gleichzeitige Zufuhr von Glykokoll vor der Säurevergiftung nicht gerettet werden konnten.

Pohl und Münzer haben auch betont, daß Ammonsalze bei Kaninchen sehr giftig wirken und daß die Symptome sich 
mit denen der Säurevergiftung decken, und deshalb bekämpfen sie Eppingers Ansicht, daß die Aminogruppe der Aminosäure eine Schutzwirkung gegen Säurevergiftung ausübt. Meine Versuche mit gleichzeitiger Zufuhr von Ammoniumacetat und Säure stehen in gutem Einklang mit den Ansichten von Pohl und Münzer, insofern, als die Tiere früher zugrunde gegangen sind als diejenigen, die nur Säure bekommen haben. Ihren Befund, daß die Tiere früher gestorben sind bei gleichzeitiger Glykokoll- und Säurezufuhr als bei Säurezufuhr allein, habe ich aber nicht bestätigen können.

Anderseits stehen meine Versuche mit denjenigen von Eppinger insoweit im Einklang, daß bei gleichzeitiger Zufuhr von Säure und Glykokoll die Ausscheidung von Ammoniak vermehrt ist.

Es ist schwer zu verstehen, warum meine Kaninchen augenscheinlich mehr widerstandsfähig gegen Säurevergiftung waren. Es ist wohl möglich, daß die Widerstandsfähigkeit bei verschiedenen Rassen und Individuen stark variiert. In dieser Hinsicht sei daran erinnert, daß nach den Angaben von Eppinger Kaninchen, die mit Blutserum gefüttert sind, der Säurevergiftung überhaupt nicht unterliegen.

\section{Schlußfolgerungen.}

Gleichzeitig subcutane Einspritzung von Glykokoll bei Säurezufuhr per Schlundsonde schützt Kaninchen nicht vor Säurevergiftung, wenn auch vielleicht der Eintritt des Todes dadurch verzögert wird.

Die gleichzeitige Verabreichung von essigsaurem Ammon mit der Säure beschleunigt den Tod der Tiere.

Bei der Säurevergiftung von Kaninchen findet gleichzeitig eine gewisse absolute Vermehrung des Ammoniaks im Harn statt. Auch der Prozentgehalt des Ammoniaks, bezogen auf den Gesamtstickstoff, nimmt zu.

Die Vermehrung des absoluten Ammoniakgehalts ist bedeutend größer, wenn Glykokoll oder essigsaures Ammon gleich-

- zeitig mit der Säure verabreicht wird. 
Es ist mir ein Bedürfnis, Herrn Geh. Rat Salkowski für seine liebenswürdige Anregung und Unterstützung bei der Arbeit meinen besten Dank zu sagen.

\section{Literatur.}

1. Salkowski, Virch. Arch:, Bd. 58.

2. Gäthgens, Centrabl. f. d. med. Wiss., 1872, S. 839.

3. Walter, Arch. f. exp. Path. u. Pharm., Bd. 7, 1877, S. 148.

4. H. Eppinger, Wien. klin. Woch., 1906, Bd. 19, Nr. 5.

5. J. Pohl und E. Münzer, Zentralbl f. Physiol., Bd. 20, Nr. 7. 